Check for updates

Cite this: RSC Adv., 2017, 7, 27564

\title{
Fabrication of PDEAEMA-based pH-responsive mixed micelles for application in controlled doxorubicin release
}

\author{
Chufen Yang, ${ }^{a}$ Jiayu Xiao, ${ }^{a}$ Weifeng Xiao, ${ }^{a}$ Wenjing Lin, (D) *a Jingrui Chen, ${ }^{a}$ \\ Quan Chen, ${ }^{\mathrm{b}}$ Lijuan Zhang, ${ }^{\mathrm{b}}{ }^{\mathrm{b}}$ Canyang Zhang ${ }^{\mathrm{c}}$ and Jianwei Guo*a
}

Co-micellization of the diblock polymers poly(ethylene glycol) methyl ether-b-poly(N,N-diethylaminoethyl methacrylate) (MPEG-PDEAEMA) and poly(ethylene glycol) methyl ether- $b$-polycaprolactone (MPEG-PCL) was carried out to avoid the complicated synthetic steps of a single polymer and enhance the drug loading contents as well as the $\mathrm{pH}$-responsive drug release performances of the polymers. The molecular weight and molecular structure of MPEG-PDEAEMA and MPEG-PCL were measured and confirmed by gel permeation chromatography (GPC) and ${ }^{1} \mathrm{H}$ NMR. With the mass ratio of MPEG-PCL and MPEGPDEAEMA set at 1:3, all three mixed micelles, MIX1 (MPEG-PCL 40 and MPEG-PDEAEMA 20 ), MIX2 (MPEG-PCL 60 and MPEG-PDEAEMA 35 ), and MIX3 (MPEG-PCL $L_{80}$ and MPEG-PDEAEMA ${ }_{50}$ ), showed good stability and excellent $\mathrm{pH}$-responsive performances according to their critical micellar concentrations (CMC), particle sizes, and zeta potentials. The doxorubicin (DOX) loading content (LC) and entrapment efficiency (EE) of the micelles were $26.79 \%$ and $63.19 \%$ (MIX1), $22.81 \%$ and $59.03 \%$ (MIX2), and $21.46 \%$ and $54.65 \%$ (MIX3), respectively. As the PDEAEMA/PCL content increased, the drug loading content decreased, which was further confirmed by dissipative particle dynamics (DPD) simulations. The results indicate that the mixed micelles developed in this study might have some advantages in improving the drug loading capacity of the polymers. The drug release profiles demonstrate that the mixed micelles have excellent ability for the controlled release of DOX, suggesting great potential for application of the micellar systems in drug delivery, especially in the area of $\mathrm{pH}$-targeted tumor treatment.

Received 18th April 2017

Accepted 9th May 2017

DOI: $10.1039 / c 7 r a 04358 a$

rsc.li/rsc-advances the most significant stimuli-responsive strategies used for tumor-targeting owing to the fact that there is a clear $\mathrm{pH}$ difference between the cellular media of tumor ( $\mathrm{pH} 5.0)$ and normal tissue ( $\mathrm{pH}$ 7.4). ${ }^{13-17}$

Generally, pH-responsive polymers, such as poly $(N, N$-dimethylaminoethyl methacrylate) (PDMAEMA), $\operatorname{poly}(N, N$ diethylaminoethyl methacrylate) (PDEAEMA), and poly( $\beta$-amino ester) (PAE), are types of polymers with $\mathrm{p} K_{\mathrm{b}}$ values for conjugate acid of about 7 . These pH-responsive polymers can easily change their conformation upon $\mathrm{pH}$ variation from a hydrophobic state at high $\mathrm{pH}\left(\mathrm{pH}>\mathrm{p} K_{\mathrm{b}}\right)$ to a hydrophilic state at low $\mathrm{pH}\left(\mathrm{pH}<\mathrm{p} K_{\mathrm{b}}\right)$, which provides considerable potential for application in tumor pH-targeted drug delivery. Among them, PDEAEMA is a brush pH-responsive polymer with a pendant tertiary amine as its responsive group and $\mathrm{p} K_{\mathrm{a}}$ of about 6.9 , and has been successfully used in anticancer drug delivery systems in the form of diblock, triblock or even more complex amphiphilic polymeric micellar structures. ${ }^{18-23}$

However, the drug loading efficiency and well-controlled drug release of these polymers still need to be improved. Especially, suitable methods to enhance the drug loading content (LC) and drug entrapment efficiency (EE), and at the same time, achieve pH-targeting drug release with a low initial 
burst release are urgently required. On one hand, in order to form stable micelles in aqueous solution, a certain proportion of hydrophilic polymer block is required for combination with the $\mathrm{pH}$-responsive DEAEMA block. On the other hand, to obtain the desired pH-responsive release performance, a sufficient amount of DEAEMA groups in the polymer is required. At the same time, to avoid immediate drug release when the PDEAEMA block changes its conformation from the hydrophobic state to hydrophilic state as the $\mathrm{pH}$ decreases, a certain amount of other hydrophobic blocks with no $\mathrm{pH}$-responsive ability is also required. However, to obtain a single polymer that self-assembles into traditional $\mathrm{pH}$-responsive polymeric micelles for a well-controlled drug release performance, complicated molecular structures and synthetic procedures are required. ${ }^{24}$ Furthermore, the drug loading content of amphiphilic polymer micelles is limited with an increase in the total hydrophobic block because a decrease in the percentage of hydrophilic block may cause hydrophilic shell thinning and instability for drug encapsulation.

Recently, the combination of two or more species of block polymers, which results in multifunctional mixed micelles, is a straightforward and potent strategy that has aroused great interest. $^{25,26}$ First, block polymers are much easier to tailor by controlled radical polymerization techniques compared to single multicomponent polymers. ${ }^{27}$ Second, the properties of mixed micelles, such as stability, particle size, $\mathrm{pH}$-responsiveness, high drug loading capacity, and well-controlled drug release, could be readily tuned by simply changing the ratios of two block polymers or altering similar substitutes.

In this work, in order to avoid the synthesis of polymers with complicated structures, simplify the synthetic steps, and improve the drug loading content as well as pH-dependent release performance, we develop mixed micelles formed by two diblock polymers for anticancer drug delivery instead of single multicomponent polymer micelles. Firstly, we synthesize two well-defined diblock amphiphilic polymers, poly(ethylene glycol) methyl ether- $b$-poly $(N, N$ diethylaminoethyl methacrylate) (MPEG-PDEAEMA) and poly(ethylene glycol) methyl ether$b$-polycaprolactone (MPEG-PCL), and then used their mixture to self-assemble pH-responsive mixed micelles. In these micelles, both MPEG-PDEAEMA and MPEG-PCL contain MPEG, which is a well-known non-immunogenic, non-antigenic and non-toxic biomaterial, as their hydrophilic block, which might improve the compatibility of the two polymers and distribute on the surface of the self-assembled micelles, thus providing a compact steric protective layer to maintain the stability of the micelles in the biological circulation. The brush structure of the PDEAEMA block, which is a good potential polymer for tumor $\mathrm{pH}$-targeting and biodegradable material, served as the $\mathrm{pH}$-responsive block, which could improve the $\mathrm{pH}$ responsiveness of the micelles to the environment. The hydrophobic PCL block, also well known for its biocompatibility and biodegradability, functions to diminish the immediate release of the micelles in acid conditions. Inside the micelles, the $\mathrm{pH}$ responsive PDEAEMA and the hydrophobic PCL blocks mix together to provide an expanded space to load hydrophobic anticancer drug, thus improving the drug loading content of the micelles. Doxorubicin (DOX), an extensively used anticancer drug, is employed as a model drug, which is encapsulated in the mixed micelles via the dialysis method. A schematic of the selfassembly and $\mathrm{pH}$-dependent drug release from the mixed polymeric micelles in aqueous solution is shown in Fig. 1. The hydrophobic interaction, which is ascribed to the PCL-PCL, PCL-PDEAEMA, PDEAEMA-PDEAEMA chains, leads to the formation of self-assembled mixed micelles. In the neutral environment of pH 7.4, the DOX-loaded mixed micelles maintain a tight and stable structure with little drug release, whereas in the tumoral weakly acidic conditions of $\mathrm{pH}$ 5.0, the encapsulated DOX could be released at an accelerated rate since the protonation of PDEAEMA leads to the swelling and disintegration of the micelles. In this work, the preparation and characterization of the polymers MPEG-PDEAEMA, MPEG-PCL, and their mixed micelles, and DOX loading content and drugrelease performance of the mixed micelles are investigated in detail.

\section{Materials and methods}

\subsection{Materials}

Methyl poly(ethylene glycol) (MPEG-OH, Mn $=5000, \mathrm{AR}), \varepsilon^{-}$ caprolactone ( $\varepsilon$-CL, 99\%), and $N, N$-diethylaminoethyl methacrylate (DEAEMA, 98\%) were obtained from Sigma-Aldrich (Germany). Triethylamine (TEA, 99\%, Sigma-Aldrich), toluene (Sigma-Aldrich), and tetrahydrofuran (THF, Sinopharm) were dried over $\mathrm{CaH}_{2}$ and distilled under nitrogen using benzophenone as a dryness indicator. 2-Bromoisobutyryl bromide (98\%) and pentamethyldiethylenetriamine (PMDETA, 99\%) were purchased from Alfa Aesar (Switzerland). Doxorubicin hydrochloride $(\mathrm{DOX} \cdot \mathrm{HCl})$ was provided by Wuhan Yuancheng Gongchuang Technology Co. Ltd. and hydrochloric acid removed before use. Pyrene (99\%), cupric bromide $\left(\mathrm{CuBr}_{2}\right)$,
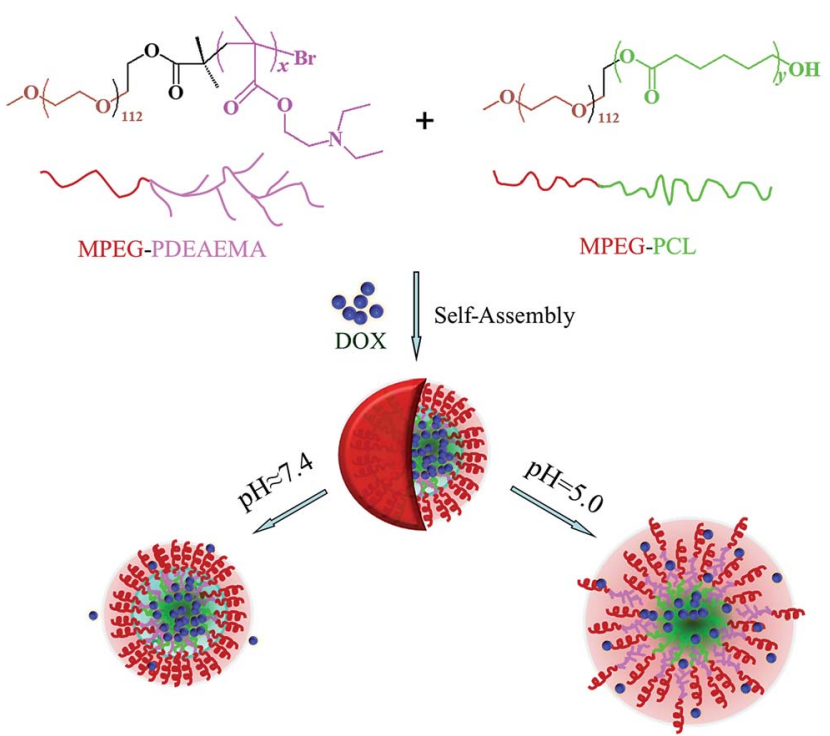

Fig. 1 Schematic of the formation of the mixed micelles and their $\mathrm{pH}$ dependent drug release. 
dimethyl sulfoxide (DMSO), stannous octoate $\left(\mathrm{Sn}(\mathrm{Oct})_{2}\right)$, and all other reagents were used without any further treatment.

\subsection{Characterizations and measurements}

${ }^{1} \mathrm{H}$ NMR spectra were obtained on a Bruker AVANCE III 400 spectrometer operating at $400 \mathrm{MHz}$ using $\mathrm{CDCl}_{3}$ as the solvent and tetramethylsilane (TMS) as the internal standard. Molecular weight $\left(M_{\mathrm{n}}\right)$ and dispersity index $\left(M_{\mathrm{w}} / M_{\mathrm{n}}\right)$ were determined by gel permeation chromatography (GPC) on an Agilent 1200 GPC equipped with an LC quant pump, polar gel columns in series, and refractive index detector, which was calibrated with a set of monodisperse PS standards using HPLC grade THF as the mobile phase at a flow rate of $1.0 \mathrm{~mL} \mathrm{~min}{ }^{-1}$ at $30^{\circ} \mathrm{C}$. The critical micelle concentration (CMC) of the MPEG-PDEAEMA and MPEG-PCL mixture was determined using a Hitachi F-4500 fluorescence spectrophotometer with an emission wavelength of $373 \mathrm{~nm}$, bandwidth of $0.2 \mathrm{~nm}$ and scanning wavelengths of $350-550 \mathrm{~nm}$ using pyrene as the fluorescence probe. The particle sizes, polydispersity index (PDI) and zeta potentials of the blank and DOX-loaded mixed micelles were measured via dynamic light scattering (DLS) with a Malvern Zetasizer Nano-S instrument. The morphology of the DOX-loaded mixed micelles was investigated via scanning electron microscopy (SEM, LEO $1530 \mathrm{VP}$, Germany). Before observation, the sample was fixed on an aluminum stub as a thin film and coated with gold.

\subsection{Synthesis of MPEG-PDEAEMA and MPEG-PCL diblock copolymers}

(1) The diblock pH-responsive amphiphilic polymer MPEGPDEAEMA was synthesized via activator regenerated by electron transfer atom transfer radical polymerization (ARGET ATRP) of DEAEMA using MPEG-Br as the macroinitiator. MPEG-Br was synthesized using the following procedure. First, MPEG-OH $(5 \mathrm{~g}, 0.1 \mathrm{mmol})$ and anhydrous THF $(30 \mathrm{~mL})$ were added successively to a $100 \mathrm{~mL}$ flame dried Schlenk flask equipped with a magnetic stirring bar under nitrogen. After the polymer dissolved, an excess amount of TEA $(0.42 \mathrm{~mL}, 0.3 \mathrm{mmol})$ was added and then the mixture was cooled to $0{ }^{\circ} \mathrm{C}$ in an ice bath. Subsequently, 2-bromoisobutyryl bromide $(0.37 \mathrm{~mL}, 0.3 \mathrm{mmol})$ was injected dropwise into the stirred mixture. After reaction for $2 \mathrm{~h}$, the mixture was warmed up to room temperature and left to stir for $24 \mathrm{~h}$. Subsequently, the mixture was passed through a neutral alumina column to remove the quaternary ammonium salts, and then precipitated by cold $\mathrm{n}$-hexane, filtered and finally collected by distillation under reduced pressure for $48 \mathrm{~h}$ to obtain MPEG-Br.

In another $100 \mathrm{~mL}$ dry Schlenk flask equipped with a magnetic stirring bar, MPEG-Br $(5.2 \mathrm{~g}, 0.1 \mathrm{mmol})$ and $\mathrm{CuBr}_{2}$ $(2.78 \mathrm{mg}, 0.012 \mathrm{mmol})$ were added under nitrogen. Then anhydrous toluene $(20 \mathrm{~mL})$, monomer DEAEMA $(4,7$ and $10 \mathrm{~mL}$, 2, 3.5 and $5 \mathrm{mmol})$ and ligand PMDETA $(75 \mu \mathrm{L}, 0.32 \mathrm{mmol})$ were added in sequence using degassed syringes. After stirring for $10 \mathrm{~min}$, an $\mathrm{Sn}(\mathrm{Oct})_{2}(103 \mu \mathrm{L}, 0.32 \mathrm{mmol})$ solution in $1 \mathrm{~mL}$ toluene was added. Then the flask was placed in an oil bath, heated to $65^{\circ} \mathrm{C}$, and maintained for $12 \mathrm{~h}$. After the reaction, the flask was removed from the oil bath and cooled to room temperature. The mixture was slowly poured into $20 \mathrm{~mL}$ THF and passed through a neutral alumina column to remove the catalyst. After removing the THF by vacuum rotary evaporation, the remaining mixture was slowly added to cold n-hexane and precipitated, filtered and finally dried under vacuum at $45^{\circ} \mathrm{C}$ for $48 \mathrm{~h}$ to obtain three diblock MPEG-PDEAEMA polymers.

(2) Diblock polymer MPEG-PCL was synthesized by the ring opening polymerization (ROP) of $\varepsilon$-CL using MPEG-OH as the macroinitiator. The typical synthetic procedure is described as follows. MPEG-OH $(2.5 \mathrm{~g}, 0.05 \mathrm{mmol})$ and $\varepsilon$-CL $(8.5,12.7$ and 17 $\mathrm{mL}, 2,3$ and $4 \mathrm{mmol}$ ) were placed in a dry $150 \mathrm{~mL}$ round bottom flask with a magnetic stirring bar. The flask was placed in an oil bath at room temperature, and then evacuated and flushed with nitrogen three times. Anhydrous THF $(20 \mathrm{~mL})$ and $\mathrm{Sn}(\mathrm{Oct})_{2}(182$ $\mu \mathrm{L}, 0.1 \mathrm{wt} \% \varepsilon$-CL) were added dropwise into the flask under nitrogen and stirred for $15 \mathrm{~min}$, and then the reaction was carried out at $80{ }^{\circ} \mathrm{C}$ for $24 \mathrm{~h}$. After the reaction, the THF was removed by vacuum rotary evaporation, and then the remaining mixture was slowly added dropwise to cold n-hexane to precipitate the product, which was dried under vacuum at $45^{\circ} \mathrm{C}$ for $48 \mathrm{~h}$, resulting in three powdery diblock MPEG-PCL polymers.

\subsection{Acid-base titration test}

To determine $\mathrm{pH}$-sensitive range of the mixture of MPEGPDEAEMA and MPEG-PCL, acid-base titration tests were performed as follows. ${ }^{28}$ MPEG-PDEAEMA and MPEG-PCL were mixed in well-defined mass ratios, then the mixtures were dissolved at a concentration of $1 \mathrm{mg} \mathrm{mL}^{-1}$ in deionized water, and the $\mathrm{pH}$ value of the solution was adjusted to around 3.0 using an $\mathrm{HCl}$ solution. Then the solution was titrated with $0.1 \mathrm{~mol} \mathrm{~L}^{-1}$ $\mathrm{NaOH}$ solution at increments of $100 \mu \mathrm{L}$. The $\mathrm{pH}$ values of the solution were monitored by an automatic titrator (Hanon T-860, Jinan, China) at room temperature.

\subsection{Critical micellar concentration (CMC) measurement}

The formation of mixed micelles self-assembled from MPEGPDEAEMA and MPEG-PCL in an aqueous phase was confirmed by the fluorescence technique using pyrene as the probe. The mixed polymers (weight ratio of MPEG-PDEAEMA to MPEGPCL: $3: 1)$ were first dissolved in deionized water $(\mathrm{pH} 7.4)$ at the concentration of $0.1 \mathrm{mg} \mathrm{mL}^{-1}$, then mixed with a pyrene solution $\left(12 \times 10^{-7} \mathrm{M}\right)$ to obtain polymer concentrations ranging from 0.0005 to $0.1 \mathrm{mg} \mathrm{mL}{ }^{-1}$. The combined solutions were equilibrated at room temperature in the dark for $48 \mathrm{~h}$ before measurement. The fluorescence excitation spectra of the polymer/pyrene solutions were used to determine the CMC values of the mixed polymers.

\subsection{Particle sizes and zeta potentials of mixed micelles}

Mixed polymeric micelles were prepared by the solvent evaporation method. $5 \mathrm{mg}$ polymer (weight ratio of MPEG-PDEAEMA to MPEG-PCL: $3: 1$ ) was dissolved in $20 \mathrm{~mL}$ acetone, followed by the dropwise addition of the solution into $50 \mathrm{~mL}$ deionized water, and continuous stirring overnight to remove acetone to obtain a $0.1 \mathrm{mg} \mathrm{mL}^{-1}$ micelle solution. $\mathrm{NaOH}$ and $\mathrm{HCl}(0.1 \mathrm{M})$ 
were used to adjust the solution $\mathrm{pH}$ value, and the particle sizes and zeta potentials of the mixed polymeric micelles at different $\mathrm{pH}$ values were measured by DLS.

\subsection{Preparation of blank and DOX-loaded mixed micelles}

Blank and DOX-loaded self-assembled micelles of the mixed polymers were prepared via the diafiltration method. For the preparation of blank micelles, the mixed polymer (MPEGPDEAEMA $15 \mathrm{mg}$ and MPEG-PCL $5 \mathrm{mg}$ ) was dissolved in $20 \mathrm{~mL}$ DMSO with vigorous stirring for $12 \mathrm{~h}$. Then the mixture was transferred to a dialysis bag (MWCO3500-4000) and dialyzed against $1 \mathrm{~L}$ deionized water for $24 \mathrm{~h}$ at room temperature. Finally the dialysate was filtered and lyophilized to obtain blank micelles in powder form.

The DOX-loaded micelles were prepared using a similar procedure. DOX-HCl (10 $\mathrm{mg})$ and the mixed polymers (MPEG-PDEAEMA $15 \mathrm{mg}$ and MPEG-PCL $5 \mathrm{mg}$ ) were dissolved in $20 \mathrm{~mL}$ DMSO. Excess TEA $(0.02 \mathrm{~mL}$ per $10 \mathrm{mg}$ DOX$\mathrm{HCl}$ ) was added to the solution to remove hydrochloride. Then the mixture was dialyzed and treated to obtain powdery DOX-loaded micelles similarly to the preparation of the blank micelles.

The DOX loading content (LC) and entrapment efficiency (EE) were adopted to evaluate the drug loading efficiency of the micelles, which were defined and calculated using the equations reported by Zhang et al. ${ }^{\mathbf{2 9 , 3 0}}$ DOX-loaded micelles (1 $\mathrm{mg}$ ) were dissolved in $10 \mathrm{~mL}$ DMSO under vigorous vortexing and were determined using a UV-2450 Shimadzu UV-vis spectrophotometer at $480 \mathrm{~nm}$, where a calibration curve was obtained with DOX-DMSO solutions with different DOX concentrations.

\subsection{In vitro release of DOX from mixed micelles}

The in vitro drug release performance of the DOX-loaded micelles formed by the mixed polymer was evaluated by comparison of the cumulative drug release percent at $\mathrm{pH} 7.4$ and 5.0 at $37^{\circ} \mathrm{C}$ (body temperature) using a Dissolution Tester (RCZ-8B, TDTF, China) and the cumulative drug release percent $\left(E_{\mathrm{r}}\right)$ calculation described in detail by Zhang et al. ${ }^{29,30}$

\subsection{Dissipative particle dynamics (DPD) simulations}

Coarse-grained simulations were performed by the DPD method to investigate the drug distribution of the drug-loaded mixed micelles, which was successfully applied in many other drug delivery systems. ${ }^{31,32}$ The polymers were divided into six types of beads: CL (green), MAA (methacrylate linked to ethylamine side chain, brown), DEA (amino ethyl side chain, pink), PEG (red), DOX (blue) and water (black). The interaction parameters were calculated by Discovery and Amorphous Cell at 298.15 K in Materials Studio 7.0 (Accelrys Inc., USA) according to our previous method..$^{30,33,34}$ A cubic simulation box of $30 \times 30$ $\times 30 r_{\mathrm{c}}{ }^{3}$ with a periodic boundary condition was applied in all three directions. The integration time step was $0.05 \mathrm{~ns}$ and the simulation steps were 100000 .

\section{Results and discussion}

\subsection{Synthesis and characterization of MPEG-PDEAEMA and MPEG-PCL polymers}

The diblock $\mathrm{pH}$-responsive amphiphilic polymer MPEGPDEAEMA was synthesized by the bromination of MPEG-OH, following ARGET ATRP using DEAEMA as the monomer. The synthetic route is shown in Fig. 2. First, the macroinitiator, MPEG-Br, was synthesized using MPEG-OH with 2-bromoisobutyryl bromide as the brominating agent and TEA as the acidbinding agent. Then the obtained MPEG-Br was using as the macroinitiator for the ARGET ATRP of DEAEMA with $\mathrm{CuBr}_{2}$ as the catalyst, $\operatorname{Sn}(\mathrm{Oct})_{2}$ as the reducing agent, and PMDETA as the ligand. The diblock polymer MPEG-PCL was synthesized by the ROP of $\varepsilon$-CL using MPEG-OH as the macroinitiator, $\varepsilon$-CL as the monomer and $\mathrm{Sn}(\mathrm{Oct})_{2}$ as the catalyst.

Three well-defined polymers of MPEG-PDEAEMA ${ }_{20}$, MPEGPDEAEMA $_{35}$ and MPEG-PDEAEMA ${ }_{50}$ were synthesized by altering the mole ratio of MPEG-Br and DEAEMA, as well as MPEG-PCL ${ }_{40}$, MPEG-PCL 60 and MPEG-PCL 80 by changing the mole ratio of MPEG-OH and $\varepsilon$-CL. The number average molecular weights $\left(M_{\mathrm{n}}\right)$ and dispersity indexes $\left(M_{\mathrm{w}} / M_{\mathrm{n}}\right)$ of these polymers were determined via GPC, and the results were shown in Table 1 and Fig. 3 . The measured $M_{\mathrm{n}, \mathrm{GPC}}$ values were very close to the $M_{\mathrm{n}, \mathrm{TH}}$ values calculated by the theory analysis from

(a)
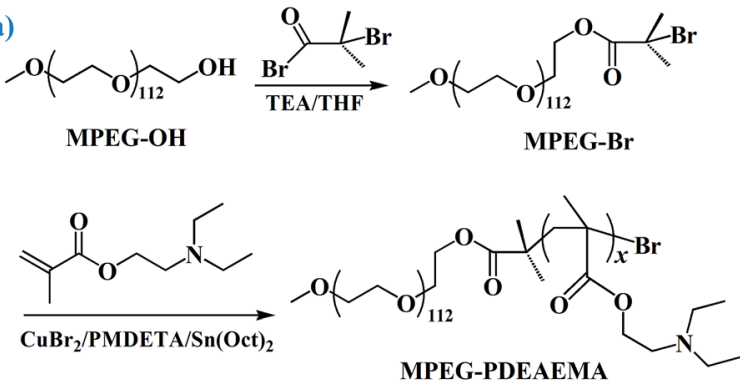

(b)

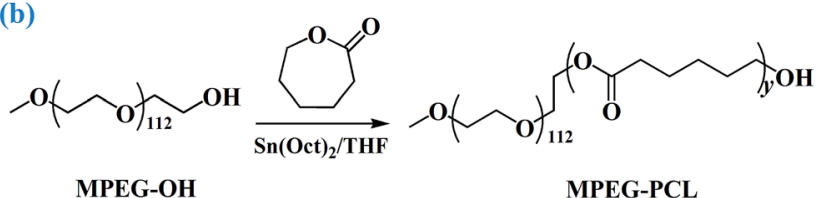

Fig. 2 Synthetic routes for MPEG-PDEAEMA and MPEG-PCL.

Table 1 GPC results of MPEG-PDEAEMA and MPEG-PCL

\begin{tabular}{llll}
\hline Sample & $M_{\mathrm{n}, \mathrm{GPC}}{ }^{a}$ & $M_{\mathrm{n}, \mathrm{TH}}{ }^{b}$ & $M_{\mathrm{w}} / M_{\mathrm{n}}{ }^{a}$ \\
\hline MPEG-PDEAEMA $_{20}$ & 9075 & 8949 & 1.04 \\
MPEG-PDEAEMA $_{35}$ & 12984 & 11624 & 1.05 \\
MPEG-PDEAEMA $_{50}$ & 14128 & 14399 & 1.06 \\
MPEG-PCL $_{40}$ & 9508 & 9560 & 1.04 \\
MPEG-PCL $_{60}$ & 12131 & 11840 & 1.03 \\
MPEG-PCL $_{80}$ & 13746 & 14120 & 1.05
\end{tabular}

${ }^{a}$ Measured by GPC in THF. ${ }^{b}$ Calculated by theory analysis from the feed ratio of monomers to initiator. 

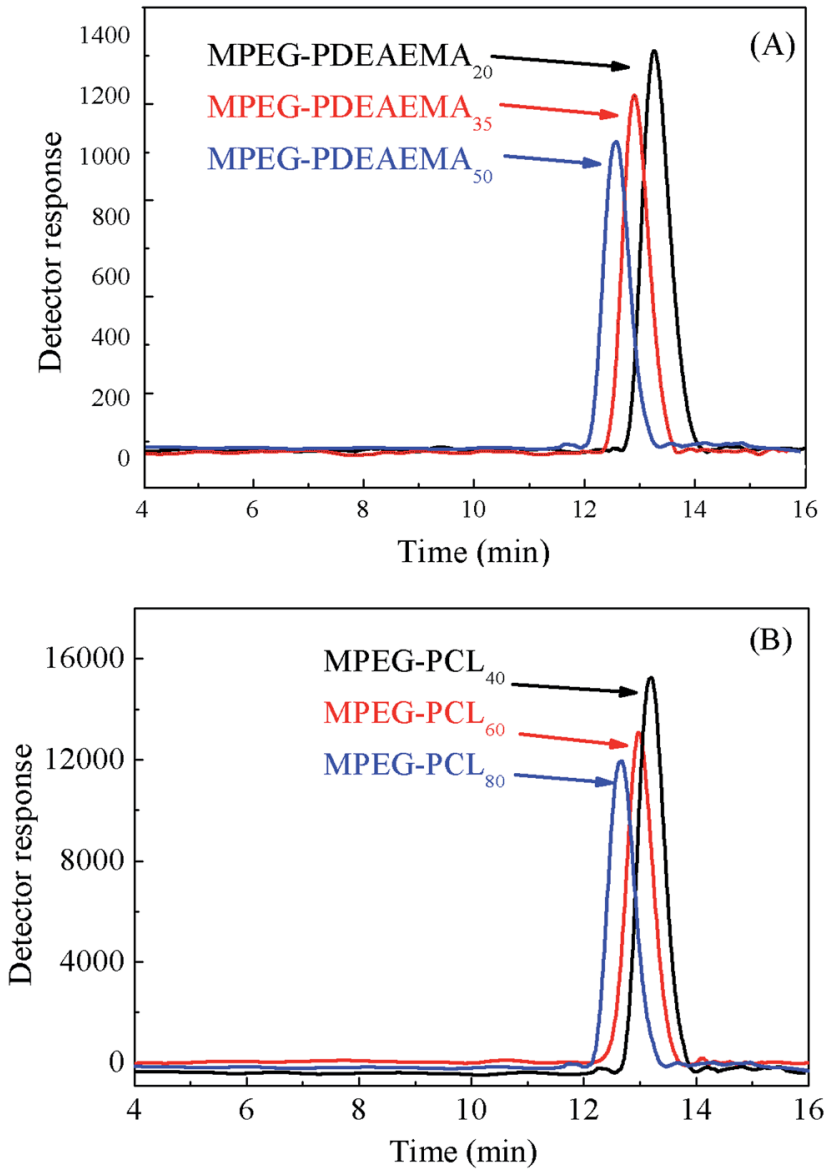

Fig. 3 GPC traces of MPEG-PDEAEMA (A) and MPEG-PCL (B).

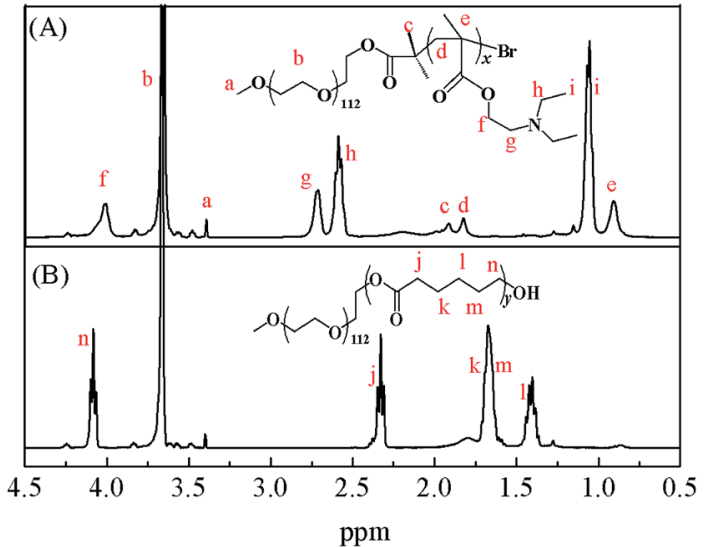

Fig. $4{ }^{1} \mathrm{H}$ NMR spectra of MPEG-PDEAEMA (A) and MPEG-PCL (B) in $\mathrm{CDCl}_{3}$.

the feed ratio of monomers to initiator. The GPC trace curves of MPEG-PDEAEMA in Fig. 3(A) and MPEG-PCL in Fig. 3(B) exhibit a narrow unimodal distribution, which suggests well a controlled ARGET ATRP and ROP process.

Representative ${ }^{1} \mathrm{H}$ NMR spectra of MPEG-PDEAEMA and MPEG-PCL are shown in Fig. 4. The signals at 3.35 (a) ppm and 3.60 (b) ppm belong to $-\mathrm{CH}_{3}$ and $-\mathrm{CH}_{2} \mathrm{CH}_{2}-$ of MPEG,
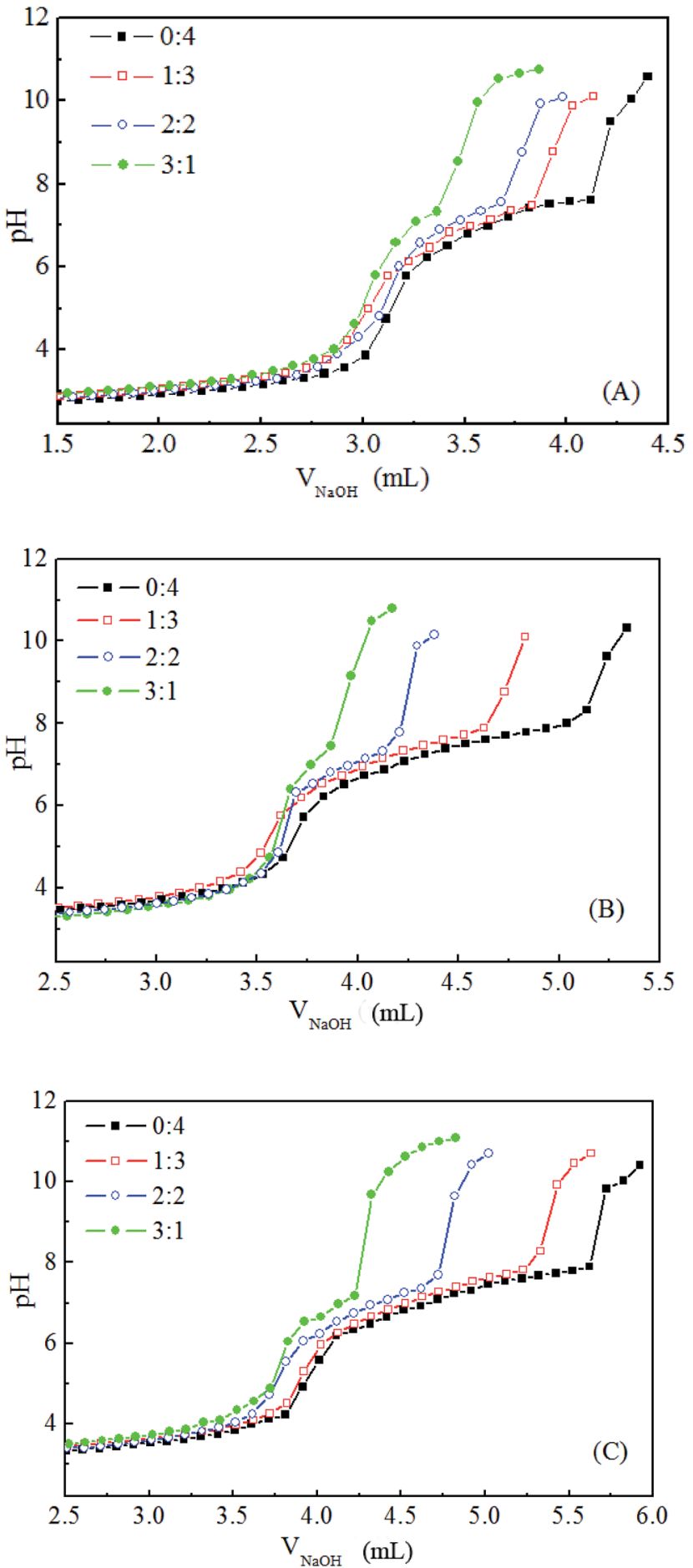

Fig. $5 \mathrm{pH}$-responsive titration curves of MIX1 (A), MIX2 (B) and MIX3 (C) in aqueous solutions with the mass ratio of MPEG-PCL and MPEGPDEAEMA at $0: 4,1: 3,2: 2$, and $3: 1$.

respectively. The signal at 1.95 (c) ppm is ascribed to $-\mathrm{C}\left(\mathrm{CH}_{3}\right)_{2}-$ of 2-bromoisobutyryl bromide, the signals of $-\mathrm{CCH}_{2-}-{ }_{-} \mathrm{CCH}_{3}-$ in the DEAEMA units appear at 1.85 (d) ppm and 0.90 (e) ppm, respectively, and the peaks at 4.05 (f) ppm, $2.70(\mathrm{~g}) \mathrm{ppm}, 2.60$ (h) ppm and 1.20 (i) ppm are the characteristic peaks of the DEAEMA unit on the side chain. The signals at 2.35 (j) ppm, 1.60 
$(\mathrm{k}, \mathrm{m}) \mathrm{ppm}, 1.40$ (l) ppm and 4.10 (n) ppm are the characteristic peaks of PCL. All the peaks corresponding to characteristic hydrogen atoms are labeled, which demonstrate that MPEGPDEAEMA and MPEG-PCL were successfully synthesized and characterized.

\section{2. $\mathrm{pH}$-responsive ranges of the mixed polymers}

The three mixed polymers discussed in this paper consist of MPEG-PDEAEMA and MPEG-PCL with similar molecular weights. In detail, MIX1 is composed of MPEG-PDEAEMA ${ }_{20}$ and MPEG-PCL ${ }_{40}$, MIX2 is composed of MPEG-PDEAEMA 35 and MPEG-PCL ${ }_{60}$ and MIX3 is composed of MPEG-PDEAEMA ${ }_{50}$ and MPEG-PCL ${ }_{80}$.

The $\mathrm{pH}$-responsive ranges of the mixed polymers were evaluated by acid-base titration, and their corresponding titration curves are shown in Fig. 5. For each mixture, the effect of different mass ratios of MPEG-PDEAEMA and MPEG-PCL on their $\mathrm{pH}$-responsive performance was determined. As expected, with the addition of $\mathrm{NaOH}$ solution, the $\mathrm{pH}$ value increased slowly and reached a plateau owing to the protonation or deprotonation of the pendant tertiary amine groups in DEAEMA when the $\mathrm{pH}$ was below or above the $\mathrm{p} K_{\mathrm{b}}$ of DEAEMA, respectively. As shown in Fig. 5, the pH-responsive regions were all in the $\mathrm{pH}$ range of 5.8-7.8 for the three types of mixed polymers. However, the $\mathrm{pH}$-responsiveness plateau feature upon the addition of $\mathrm{NaOH}$ is influenced by the PDEAEMA segment length, as well as the mass ratio of MPEG-PDEAEMA to MPEGPCL. The pH-responsive plateau for $\mathrm{NaOH}$ addition in Fig. 5(C) is wider than that in Fig. 5(B), and Fig. 5(B) wider than that in Fig. 5(A), which demonstrate that the pH-responsive performance of MIX3 is superior to MIX2, and MIX2 superior to MIX1.

On the other hand, good $\mathrm{pH}$-responsive properties were exhibited in aqueous solution when the mass ratios of MPEGPCL to MPEG-PDEAEMA were $0: 4$ and $1: 3$, and fairly $\mathrm{pH}$ responsive capability was obtained at a mass ratio $2: 2$, but at a mass ratio of $3: 1$, poor $\mathrm{pH}$-responsive behavior was displayed. Herein, to prepare good $\mathrm{pH}$-responsive micelles, the

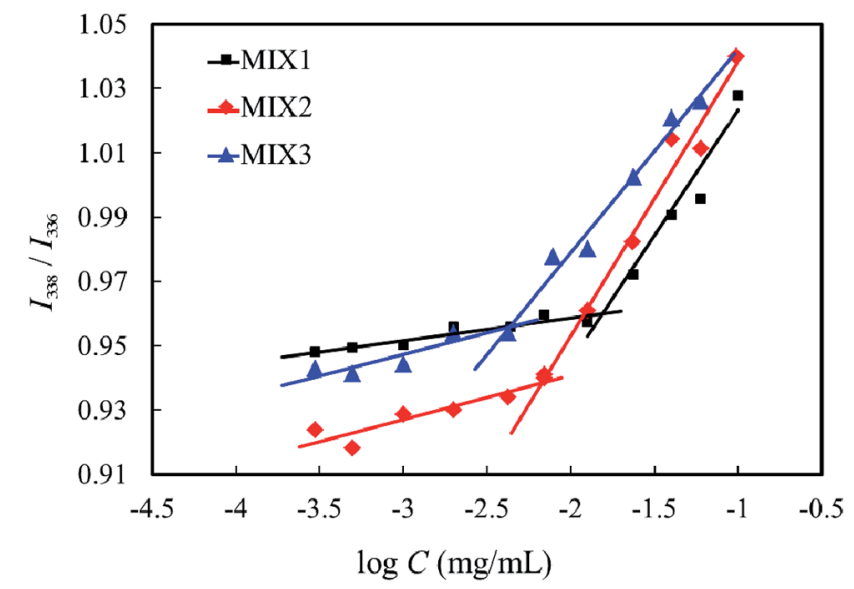

Fig. $6 I_{338} / I_{336}$ ratios of pyrene in neutral aqueous solution of the mixed polymers at various concentrations. mass ratio of MPEG-PCL and MPEG-PDEAEMA in the mixed polymers was set at $1: 3$.

\subsection{CMC values}

The CMC value is one of the most important characters of amphiphilic polymers, which is related to the thermodynamically stability of drug-loaded micelles and affects the initial release of the drug when introduced into the bloodstream by intravenous administration. The CMC values of the three mixed polymers, MIX1, MIX2 and MIX3, with the mass ratio of MPEGPCL and MPEG-PDEAEMA set at $1: 3$, were determined by florescence spectroscopy using pyrene as the probe, and the results are shown in Fig. 6. The CMC values of MIX1, MIX2, and MIX3 in neutral aqueous solution are $12.59 \mathrm{mg} \mathrm{L}^{-1}, 6.31 \mathrm{mg} \mathrm{L}^{-1}$ and $4.47 \mathrm{mg} \mathrm{L}^{-1}$, respectively, which indicate that the stability of the micelles formed by MIX3 is superior to MIX2, and MIX2 superior to MIX1. It is well known that a longer hydrophobic block in copolymers results in a lower CMC value, hence the mixed polymers with a longer PCL length and longer PDEAEMA had a lower CMC. It is concluded that the micelles formed by MIX3 are more stable than that of MIX2, and that of MIX2 more stable than that of MIX1.

\subsection{Particle sizes and zeta potential of the blank mixed micelles}

The particle sizes and zeta potentials of the blank mixed micelles formed by MIX1, MIX2 and MIX3 were detected by dynamic light scattering (DLS, Fig. 7 and 8, respectively). As shown in Fig. 7, when the $\mathrm{pH}$ value decreased from 10.0 to 8.0, the particle sizes of the blank micelles were almost unchanged. This is because hardly any of the PDEAEMA block was protonated in this $\mathrm{pH}$ value range, which lead to the aggregation of the mixed polymers micelles in a compact structure and the formation of isolated nanoparticles. When the $\mathrm{pH}$ value decreased continuously from 7.0 to 5.0, the particle sizes of the micelles increased obviously because the tertiary amine groups of the PDEAEMA block were gradually protonated and the

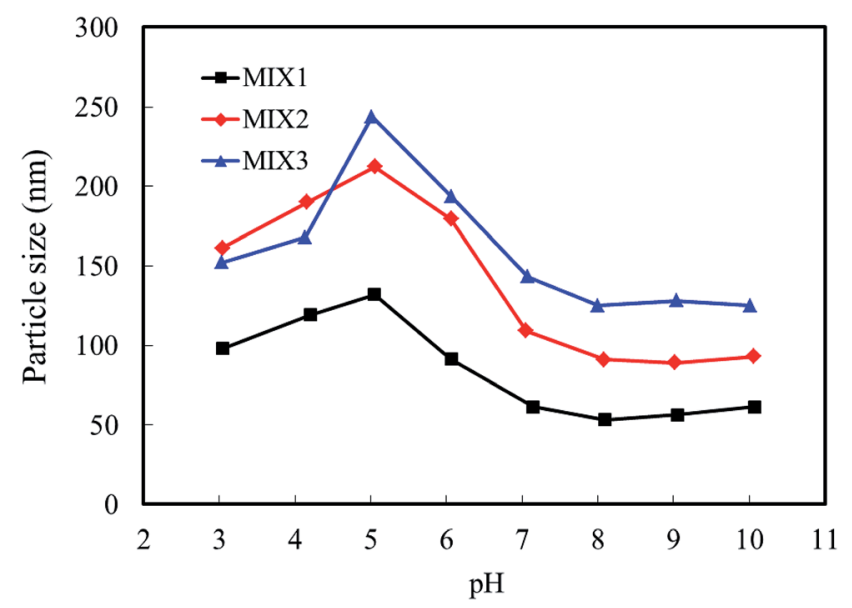

Fig. 7 Particle size of the mixed polymeric micelles dependence on $\mathrm{pH}$ value in aqueous solution. 


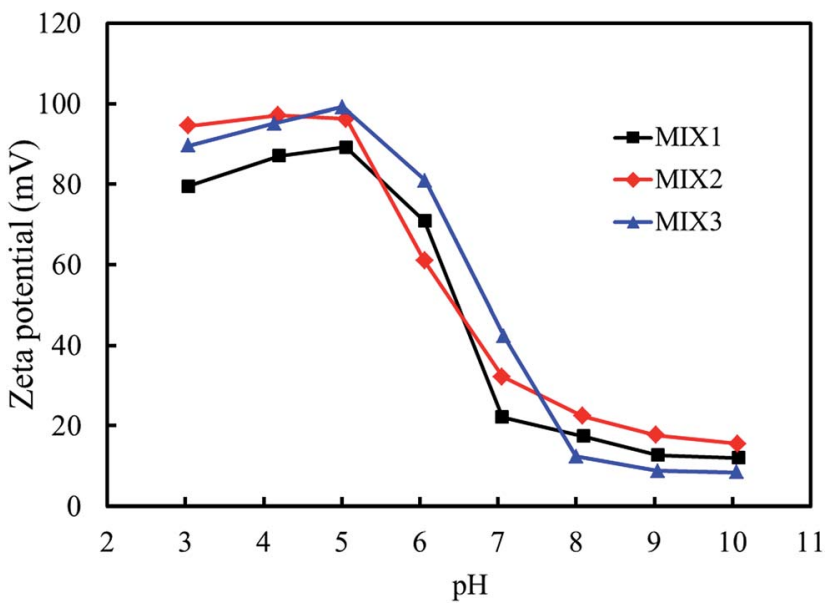

Fig. 8 Zeta potential of the mixed polymeric micelles dependence on $\mathrm{pH}$ value in aqueous solution.

Table 2 Size, polydispersity index, LC and EE of the DOX-loaded mixed micelles

\begin{tabular}{lllll}
\hline Sample & $\operatorname{Size}^{a}(\mathrm{~nm})$ & $\mathrm{PDI}^{a}$ & $\mathrm{LC}^{a}(\%)$ & $\mathrm{EE}^{a}(\%)$ \\
\hline MIX1 & 213 & 0.23 & 26.79 & 63.19 \\
MIX2 & 278 & 0.31 & 22.81 & 59.03 \\
MIX3 & 293 & 0.34 & 21.46 & 54.65
\end{tabular}

${ }^{a}$ Measured at a feed ratio of $10 \mathrm{mg}$ DOX/20 mg mixed polymer.

transformation of the polymer from hydrophobic to hydrophilic, resulting in a loose and swollen micellar structures. When the $\mathrm{pH}$ value decreased from 5.0 to 3.0, the particle sizes gradually decreased, which is attributed to the PDEAEMA block being fully protonated and becoming a hydrophilic polymer, which resulted in a decrease in the aggregation number of the mixed polymers or even slight dissociation of the micelles. Comparing the particle sizes of the micelles formed by MIX1, MIX2 and MIX3, which formed large multimolecular micelles, it was found that a more prominent $\mathrm{pH}$ dependent change was achieved in MIX2 and MIX3, which indicates their better $\mathrm{pH}$ responsiveness than MIX1.

With respect to zeta potential, Fig. 8 displays that the zeta potentials of the blank mixed micelles depend on the $\mathrm{pH}$ value. As the $\mathrm{pH}$ value decreased from 10.0 to 8.0, the zeta potentials of the micelles remained almost constant. As the $\mathrm{pH}$ dropped sequentially from 7.0 to 5.0, the zeta potentials increased rapidly, which is attributed to the continuous protonation of the PDEAEMA block. As the pH decreased from 5.0 to 3.0, the zeta potentials decreased slightly. Briefly, the zeta potentials of the micelles depended on the $\mathrm{pH}$ values, which exhibit almost the same tendency with the particle sizes, thus indicating that the micelles have pH-responsive self-assembly behavior.

\subsection{Characterization of DOX-loaded mixed micelles}

The particle sizes and polydispersity index (PDI) of the DOXloaded mixed micelles were detected by dynamic light scattering (DLS) and SEM, respectively. LC and EE were determined by UV-vis spectrophotometry. As shown in Table 2, DOX-loading resulted in multimeric MIX1, MIX2, and MIX3 micelles with a hydrodynamic diameter $D_{\mathrm{h}}$ of 213, 278 and $293 \mathrm{~nm}$ (by DLS) and dry particle size of 220, 250 and $280 \mathrm{~nm}$ (by SEM, Fig. 9), respectively. The DOX-loaded micelles formed by MIX3 showed bigger particle sizes compared to that of MIX1 and MIX owing to the increasing length of the PDEAEMA and PCL chains in the mixed polymers, where the longer hydrophobic chains aggregated into much bigger multimolecular micelles with larger particle sizes. Their PDI were all less than 0.4 , which indicates a narrow unimodal distribution and good physical performance of the micelles.

We found that the LC and EE of the micelles formed by MIX1 were $26.79 \%$ and $63.19 \%$, which are much higher than that of MIX2 (22.81\% and 59.03\%) and MIX3 (21.46\% and 54.65\%), respectively. Using the same concentrations as the actual experiment (volume fraction of mixed micelles 10\%, DOX 5\%, and water $85 \%$ ), the DOX loading capacity and detailed DOX distribution of the mixed micelles were tracked in simulations, which predicted that DOX tended to distribute in the mixed inner core formed by hydrophobic PCL and $\mathrm{pH}$-sensitive PDEAEMA chains owing to the hydrophobic interaction

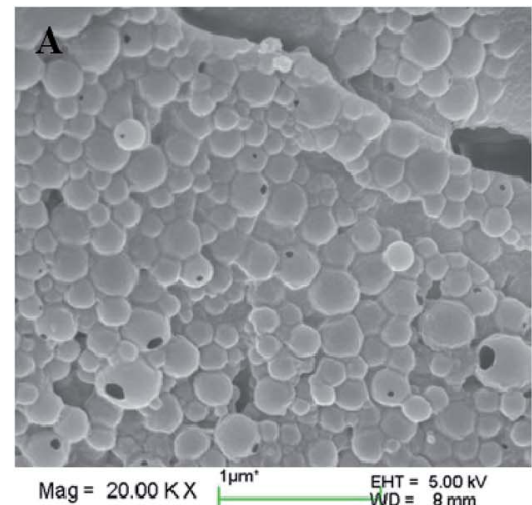

Mag $=20.00 \mathrm{KX} \begin{aligned} E H T & =5.00 \mathrm{kV} \\ & =8 \mathrm{~mm}\end{aligned}$

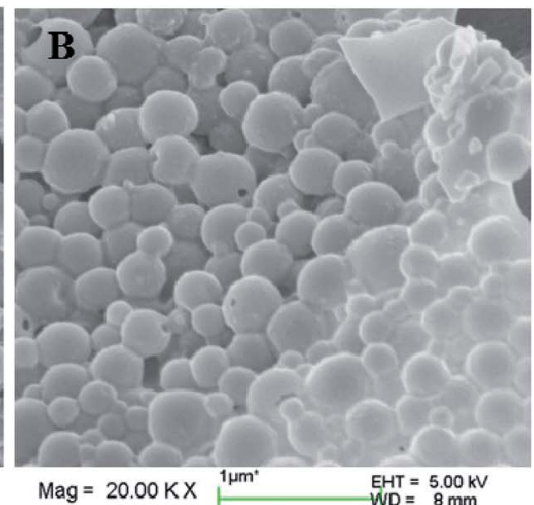

$\mathrm{Mag}=20.00 \mathrm{KX}{ }^{1 \mu \mathrm{m}^{*}} \quad \begin{aligned} & E H T=5.00 \mathrm{kV} \\ & W D=8 \mathrm{~mm}\end{aligned}$

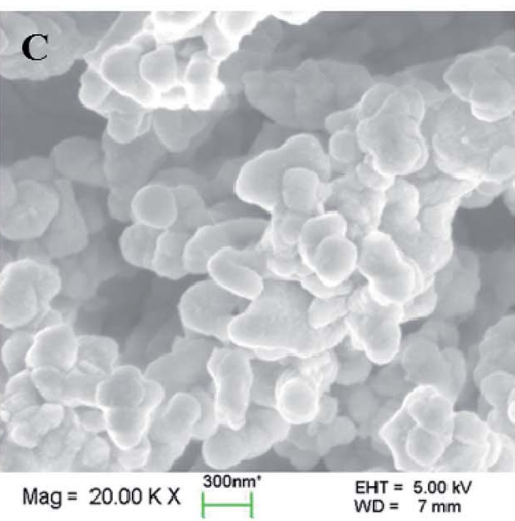

Fig. 9 SEM images of the DOX-loaded MIX1 (A), MIX2 (B) and MIX2 (C) micelles. 


\section{Full-section views Cross-section views}

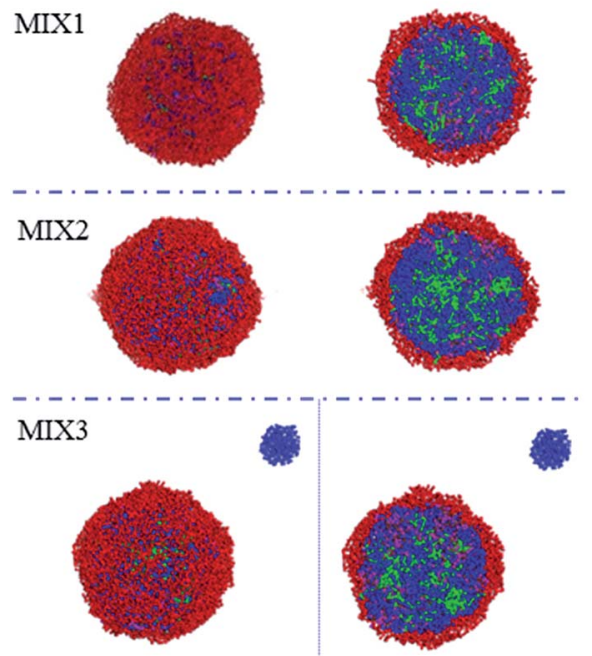

Fig. 10 Full-section and cross-section views of the DOX-loaded mixed micelles.

between DOX and PCL/PDEAEMA. As the PCL/PDEAEMA chains of the polymers increased in length, the capacity of DOX decreased, which is accord with the above experimental results (Fig. 10). This might be due to the fact that the proportion of hydrophilic PEG is relatively smaller and thinner when the amount of PCL/PDEAEMA chains increase but the same length of PEG chains is maintained, thus forming MIX3 with a loose shell and series of cracks which provide channels for the diffusion of DOX into the water solution even though it was encapsulated. Therefore, the capacity of the encapsulated DOX had to be reduced in order to make PEG hydrophilic enough to distribute in the micelle surface and provide a compact steric protective layer to maintain the stability of the micelles. Nevertheless, the drug LC and EE of the micelles formed by these three mixed micelles are still superior to many other $\mathrm{pH}$ responsive micelles formed by single amphiphilic polymer systems. ${ }^{35,36}$

Briefly, an excellent drug loading capacity is not only determined by the high drug loading capacity of the hydrophobic block, but also the protective effect of the hydrophilic shell. By fine-tuning and well controlling the proportion of hydrophilic and hydrophobic components, mixed micelles would provide some advantages for the design of micelles with expected drug loading capacities.

\subsection{In vitro DOX release of DOX-loaded mixed micelles}

To evaluate the effects of the $\mathrm{pH}$-responsive behavior on controlled drug delivery, the in vitro DOX release performances of the DOX-loaded micelles formed by MIX1, MIX2 and MIX3 were determined under the physiological condition ( $\mathrm{pH} 7.4)$ as well as in a slightly acidic environment (pH 5.0) (Fig. 11). It was found that the DOX release rates of the micelles formed by MIX1, MIX2 and MIX3 were all pH-dependent, and their release rates were accelerated when the $\mathrm{pH}$ values decreased from 7.4 to 5.0.

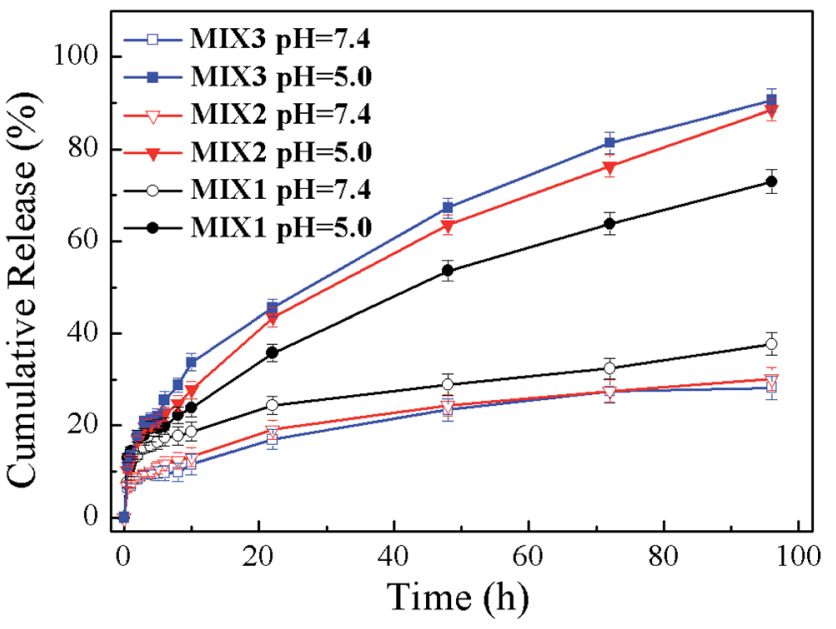

Fig. 11 DOX release profiles of the DOX-loaded mixed micelles at $\mathrm{pH}$ 7.4 and $\mathrm{pH} 5.0$.

At a pH of 7.4 , only about $10 \%$ of DOX from the micelles formed by MIX2 and MIX3 was released in $12 \mathrm{~h}$, then the release rate increased slowly and tended to remain constant, where about $15 \%$ of DOX was released in $24 \mathrm{~h}$, which is less than $25 \%$ after $96 \mathrm{~h}$, which indicates that the micelles could remain stable at $\mathrm{pH} 7.4$ and the drug was well protected with a low DOX release from the micelles in the blood circulation.

At pH 5.0, the DOX release rate was increased obviously since the protonation of the tertiary amine groups of PDEAEMA led to the swelling of the micelles. The cumulative release of the micelles formed by MIX2 and MIX3 was about 20\% after $6 \mathrm{~h}$, around $40 \%$ after $24 \mathrm{~h}$, and nearly $90 \%$ after 96, which demonstrate the excellent pH-responsive sensitivity of MIX2 and MIX3. However, the pH-responsiveness of MIX1 was slightly poor in comparison because of the fewer $\mathrm{pH}$-responsive chains of PDEAEMA in the mixed micelles.

The semi-empirical equation established by Ritger and Peppas was used to explore the mechanism of drug release from the micelles (Fig. 12)..$^{37-39}$ The release of DOX was divided into two stages, the first was from 0 to $10 \mathrm{~h}$ and the other from 10 to $96 \mathrm{~h}$. The fitting parameters, including the release exponent $n$, rate constant $k$, and correlation coefficient $R^{2}$, are shown in Table 3. Each stage displayed good linearity. The $n$ values of MIX1, MIX2, and MIX3 obtained at pH 7.4 were lower than 0.43 in both stages due to the combined effect of diffusion and erosion control. When the $\mathrm{pH}$ decreased to 5.0, the $n$ values of MIX1, MIX2, and MIX3 in the first stage were still lower than 0.43 , which corresponds to the combination of diffusion and erosion control. Since all the PDEAEMA was protonated after $10 \mathrm{~h}$, the micelles had swollen into a looser structure, and the DOX release was mainly controlled by an anomalous transport mechanism, with the $n$ values of the second stage of MIX1, MIX2, and MIX3 being $0.48,0.48$ and 0.53 , respectively. The $k$ values at both stages increased as the $\mathrm{pH}$ decreased from 7.4 to 5.0, which suggest that the release rates accelerate at low $\mathrm{pH}$, in accord with the in vitro release experiment results. Moreover, the $k$ values of MIX3 were higher than that of MIX1 and MIX2 at 

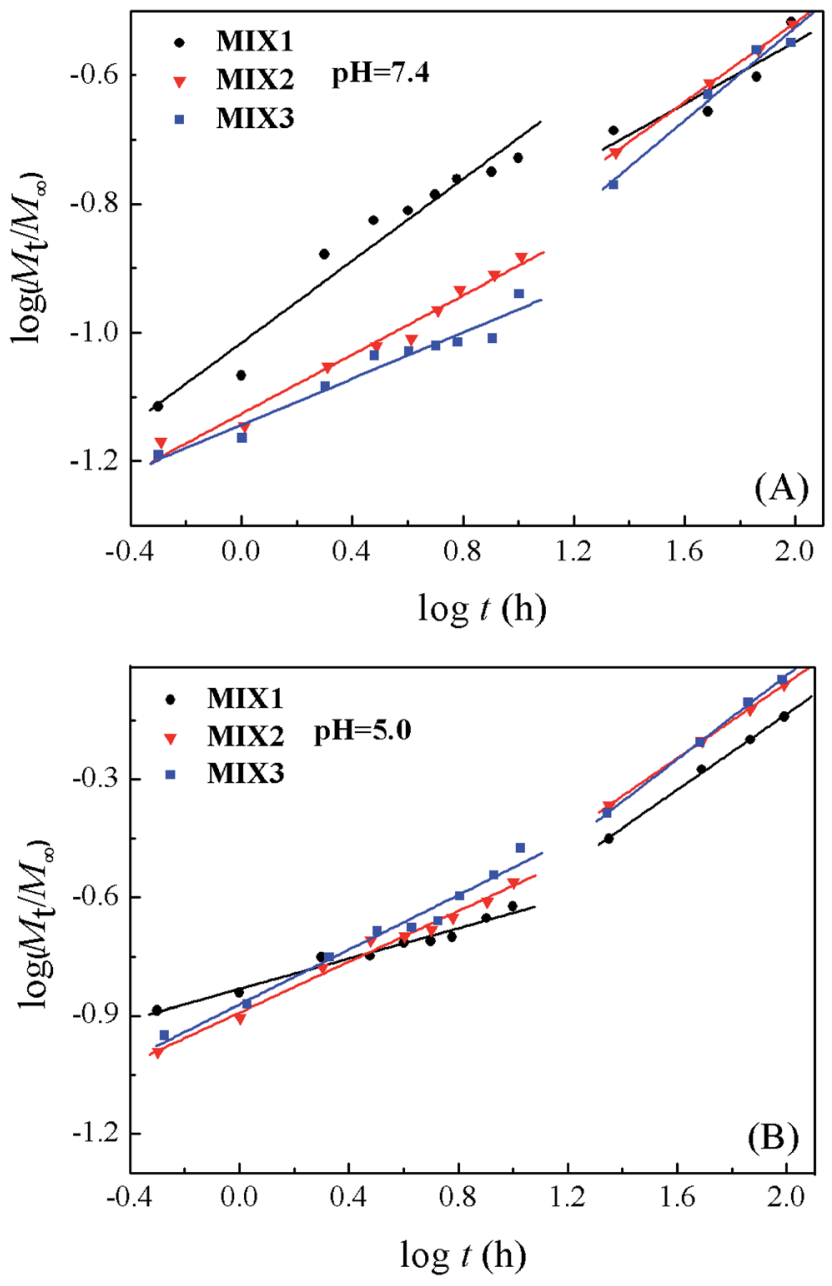

Fig. 12 Plots of $\log \left(M_{t} / M_{\infty}\right)$ against $\log t$ for DOX release from the mixed micelles at $\mathrm{pH} 7.4$ and 5.0.

Table 3 Fitting parameters of the DOX release model at $\mathrm{pH} 7.4$ and 5.0

\begin{tabular}{llllllll}
\hline $\mathrm{pH}$ & Matrix & $n_{1}{ }^{a}$ & $k_{1}{ }^{a}$ & $R_{1}{ }^{2}$ & $n_{2}{ }^{b}$ & $k_{2}{ }^{b}$ & $R_{2}{ }^{2}$ \\
\hline 7.4 & MIX1 & 0.318 & 0.096 & 0.945 & 0.270 & 0.101 & 0.943 \\
& MIX2 & 0.230 & 0.075 & 0.972 & 0.309 & 0.074 & 1.000 \\
& MIX3 & 0.180 & 0.072 & 0.942 & 0.361 & 0.056 & 0.965 \\
5.0 & MIX1 & 0.193 & 0.147 & 0.969 & 0.482 & 0.081 & 0.997 \\
& MIX2 & 0.193 & 0.130 & 0.986 & 0.479 & 0.099 & 0.999 \\
& MIX3 & 0.347 & 0.138 & 0.972 & 0.532 & 0.100 & 0.996
\end{tabular}

${ }^{a}$ The first stage is $0-10 \mathrm{~h}^{b}$ The second stage is $10-96 \mathrm{~h}$.

pH 5.0 owing to its structural advantage of a longer PDEAEMA block.

To sum up, the drug release rates of DOX from the mixed micelles significantly accelerated as the $\mathrm{pH}$ decreased and PDEAEMA content increased. Thus, the pH-responsive release profiles of the mixed polymer micelles are clearly in agreement with the requirements of anticancer drug administration, providing slight leakage at normal tissues and accelerated release behavior at tumor cells (endosomal and lysosomal), which could be used as latent vehicles for the delivery of different types of hydrophobic drugs with controlled release behavior.

\section{Conclusions}

Well-defined MPEG-PDEAEMA and MPEG-PCL polymers were successfully synthesized and characterized via $1 \mathrm{H}$ NMR and GPC, and used to prepare $\mathrm{pH}$-responsive mixed micelles for anticancer drug delivery. The drug LC and EE of the mixed micelles achieved were $26.79 \%$ and $63.19 \%$ (MIX1), $22.81 \%$ and $59.03 \%$ (MIX2), and $21.46 \%$ and 54.65\% (MIX3), respectively, measured at a feed ratio of $10 \mathrm{mg}$ DOX to $20 \mathrm{mg}$ mixed polymer, which indicate that the mixed micelles developed in this paper might contribute to the improvement of the drug loading capacity of polymers. The drug release profiles demonstrated that the swelling of PDEAEMA in the micelles at low pH accelerated the drug diffusion into aqueous solution. Overall, this study presents strategy idea of combining the functional advantages of two simple diblock polymers to optimize selfassembled polymeric micelles for cancer targeted therapy.

\section{Acknowledgements}

Financial support from the National Natural Science Foundation of China (No. 21676058 and No. 21476051) and the Natural Science Foundation of Guangdong Province (No. 2016A030313694) was gratefully acknowledged.

\section{References}

1 A. Mandal, R. Bisht, I. D. Rupenthal and A. K. Mitra, J. Controlled Release, 2017, 248, 96-116.

2 M. W. Amjad, P. Kesharwani, M. C. I. Mohd Amin and A. K. Iyer, Prog. Polym. Sci., 2017, 64, 154-181.

3 H. S. Oberoi, N. V. Nukolova, A. V. Kabanov and T. K. Bronich, Adv. Drug Delivery Rev., 2013, 65, 1137-1152.

4 Y. Q. Yang, X. D. Guo, W. J. Lin, L. J. Zhang, C. Y. Zhang and Y. Qian, Soft Matter, 2012, 8, 454-464.

5 S. Yin, L. Chang, T. Li, G. Wang, X. Gu and J. Li, RSC Adv., 2016, 6, 105957-105968.

6 K.-Y. Lee, Y.-T. Chiu and C.-L. Lo, RSC Adv., 2015, 5, 8382583836.

7 J. Zhuang, M. R. Gordon, J. Ventura, L. Li and S. Thayumanavan, Chem. Soc. Rev., 2013, 42, 7421-7435.

8 E. G. Kelley, J. N. L. Albert, M. O. Sullivan and I. I. I. T. H. Epps, Chem. Soc. Rev., 2013, 42, 7057-7071.

9 J. Hu, G. Zhang and S. Liu, Chem. Soc. Rev., 2012, 41, 59335949.

10 H. Wei, R. X. Zhuo and X. Z. Zhang, Prog. Polym. Sci., 2013, 38, 503-535.

11 L. Wang, J. Luan, L. Du, L. Li, J. Liu, Z. Liu and R. Zhuo, RSC Adv., 2016, 6, 111161-111169.

12 G. Yan, J. Wang, L. Hu, X. Wang, G. Yang, S. Fu, X. Cheng, P. Zhang and R. Tang, Acta Biomater., 2017, 51, 363-373. 
13 S. Bazban-Shotorbani, M. M. Hasani-Sadrabadi, A. Karkhaneh, V. Serpooshan, K. I. Jacob, A. Moshaverinia and M. Mahmoudi, J. Controlled Release, 2017, 253, 46-63.

14 R. Tang, W. Ji, D. Panus, R. N. Palumbo and C. Wang, J. Controlled Release, 2011, 151, 18-27.

15 Y. Li, M. Leng, M. Cai, L. Huang, Y. Chen and X. Luo, Colloids Surf., B, 2017, 154, 397-407.

16 C. Y. Zhang, Y. Q. Yang, T. X. Huang, B. Zhao, X. D. Guo, J. F. Wang and L. J. Zhang, Biomaterials, 2012, 33, 6273-6283.

17 A. Mozhi, I. Ahmad, C. I. Okeke, C. Li and X.-J. Liang, RSC Adv., 2017, 7, 12886-12896.

18 H. Liu, C. Li, H. Liu and S. Liu, Langmuir, 2009, 25, 47244734.

19 S. H. Tang, X. Q. Huang, X. L. Chen and N. F. Zheng, Adv. Funct. Mater., 2010, 20, 2442-2447.

20 Y. Q. Yang, B. Zhao, Z. D. Li, W. J. Lin, C. Y. Zhang, X. D. Guo, J. F. Wang and L. J. Zhang, Acta Biomater., 2013, 9, 76797690.

21 C. Y. Zhang, W. S. Wu, N. Yao, B. Zhao and L. J. Zhang, RSC Adv., 2014, 4, 40232-40240.

22 W. Lin, S. Nie, D. Xiong, X. Guo, J. Wang and L. Zhang, Nanoscale Res. Lett., 2014, 9, 243.

23 P. D. Topham, J. R. Howse, O. O. Mykhaylyk, S. P. Armes, R. A. L. Jones and A. J. Ryan, Macromolecules, 2006, 39, 5573-5576.

24 X. Huang, Y. Xiao and M. Lang, J. Colloid Interface Sci., 2011, 364, 92-99.

25 A. B. Ebrahim Attia, Z. Y. Ong, J. L. Hedrick, P. P. Lee, P. L. R. Ee, P. T. Hammond and Y.-Y. Yang, Curr. Opin. Colloid Interface Sci., 2011, 16, 182-194.

26 Y.-C. Chen, C.-L. Lo, Y.-F. Lin and G.-H. Hsiue, Biomaterials, 2013, 34, 1115-1127.
27 C.-F. Mu, P. Balakrishnan, F.-D. Cui, Y.-M. Yin, Y.-B. Lee, H.-G. Choi, C. S. Yong, S.-J. Chung, C.-K. Shim and D.-D. Kim, Biomaterials, 2010, 31, 2371-2379.

28 Q. Chen, W. Lin, H. Wang, J. Wang and L. Zhang, RSC Adv., 2016, 6, 68018-68027.

29 W. Lin, S. Nie, Q. Zhong, Y. Yang, C. Cai, J. Wang and L. Zhang, J. Mater. Chem. B, 2014, 2, 4008-4020.

30 W. J. Lin, S. Y. Nie, Q. Chen, Y. Qian, X. F. Wen and L. J. Zhang, AIChE J., 2014, 60, 3634-3646.

31 X. D. Guo, L. J. Zhang, Z. M. Wu and Y. Qian, Macromolecules, 2010, 43, 7839-7844.

32 Y. Wang, J. W. Ren, C. Y. Zhang, M. Chan He, Z. M. Wu and X. D. Guo, RSC Adv., 2016, 6, 101323-101333.

33 S. Y. Nie, Y. Sun, W. J. Lin, W. S. Wu, X. D. Guo, Y. Qian and L. J. Zhang, J. Phys. Chem. B, 2013, 117, 13688-13697.

34 Y. Li, G. Xu, Y. Luan, S. Yuan and Z. Zhang, Colloids Surf., A, 2005, 257-258, 385-390.

35 S. Y. Moon, Y. S. Choi, J.-K. Cho, M. Yu, E. Lee, K. M. Huh, D. H. Lee, J.-H. Kim and H. C. Kang, RSC Adv., 2016, 6, 15558-15576.

36 J. Zhao, J. Liu, S. Xu, J. Zhou, S. Han, L. Deng, J. Zhang, J. Liu, A. Meng and A. Dong, ACS Appl. Mater. Interfaces, 2013, 5, 13216-13226.

37 C. R. Young, C. Dietzsch, M. Cerea, T. Farrell, K. A. Fegely, A. Rajabi-Siahboomi and J. W. McGinity, Int. J. Pharm., 2005, 301, 112-120.

38 P. L. Ritger and N. A. Peppas, J. Controlled Release, 1987, 5, 23-36.

39 J. Siepmann and A. Göpferich, Adv. Drug Delivery Rev., 2001, 48, 229-247. 